\section{Adiponectin-good for the mother and her offspring?}

Infusing obese pregnant mice with adiponectin restores maternal insulin sensitivity, protects placental function and reduces excessive fetal growth, according to new research.

Fetuses exposed to an obese intrauterine environment are at increased risk of obesity in childhood. "This creates a vicious, detrimental cycle of intrauterine transmission of metabolic disease from the mother to her children," explains corresponding author Irving Aye. Once established, obesity and the metabolic syndrome are difficult to treat, which led Aye and colleagues to consider preventive approaches using interventions during pregnancy in mothers with obesity. Adiponectin infusion in lean pregnant mice reduces fetal growth, so the investigators hypothesized that adiponectin could limit the fetal overgrowth that can result from maternal obesity.

The researchers fed female mice either a standard chow diet or a high-fat diet that induced obesity and insulin resistance and mated them with chow-fed males. The obese dams were infused with adiponectin or phosphate-buffered saline (PBS) for 4 days at the end of pregnancy, which covered $70 \%$ of total fetal growth.

In the obese dams, treatment with adiponectin reduced insulin resistance and circulating levels of cholesterol compared with PBS treatment. In addition, adiponectin treatment prevented the overgrowth of fetuses and the increase in glucose levels that was observed in the PBS-treated obese dams. Interestingly, the protective effects of adiponectin occurred independently of changes in maternal fat mass.

Next, the researchers investigated the impact of adiponectin treatment on the regulation of placental nutrient transport in vivo. They showed that placental nutrient transport increased dramatically as a result of maternal obesity. By contrast, placental function in

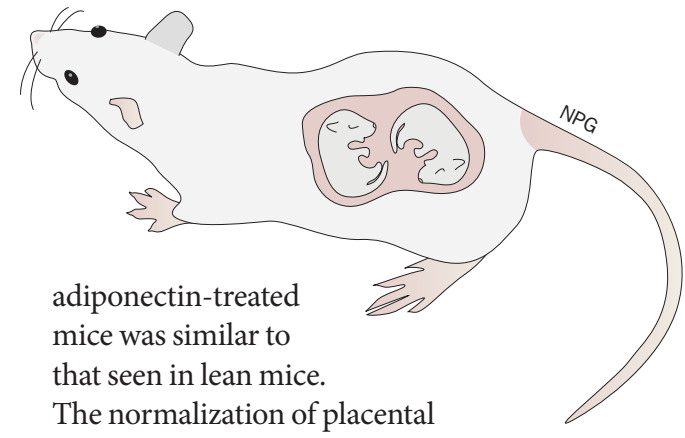

function was associated with reduced activation of the mTORC1 signalling pathway, which was highly active in the placentas of untreated obese mice.

"The next step is to study the offspring of obese mice and obese mice supplemented with adiponectin ... to demonstrate that treatment also prevents the long-term adverse consequences of maternal obesity," comments author Fredrick Rosario. "The preliminary results are very promising.”

\section{Hugh Thomas}

Original article Aye, I. L. M. et al. Adiponectin supplementation in pregnant mice prevents the adverse effects of maternal obesity on placental function and fetal growth. Proc. Natl Acad. Sci. USA doi:10.1073/ pnas. 1515484112 